\title{
POTASSIUM CONTROL IN CHRONIC KIDNEY DISEASE: IMPLICATIONS FOR NEUROMUSCULAR FUNCTION
}

Ria Arnold ${ }^{1}$, Tim J Pianta ${ }^{2}$ Bruce A. Pussell ${ }^{3,4}$, Zoltan Endre ${ }^{4}$, Matthew C. Kiernan ${ }^{5}$ and Arun V. Krishnan $^{3}$

(1) School of Medical Sciences, UNSW Sydney (2) Northern Clinical School, University of Melbourne, Melbourne (3) Prince of Wales Clinical School, UNSW Sydney (4) Department of Nephrology Prince of Wales Hospital, Sydney (5) Brain and Mind Centre, University of Sydney, Sydney.

Author Contributions: RA contributed to the conception and design of the review, analysed and interpreted the data, drafted and revised it for critically for important intellectual content and approved the final version for publication. TJP contributed to drafting, analysis and interpretation of data, revised it for critically for important intellectual content and approved the final version for publication: BAP contributed to the conception and design of the review revised it for critically for important intellectual content and approved the final version for publication. ZE revised it for critically for important intellectual content and approved the final version for publication. MCK contributed to the conception and design of the review revised it for critically for important intellectual content and approved the final version for publication. AVK contributed to the conception and design of the review revised it for critically for important intellectual content and approved the final version for publication.

\section{Correspondence to:}

Professor Arun Krishnan

Prince of Wales Clinical School

University of New South Wales, Sydney NSW 2052 Australia

Ph: +61 293822414

Email: arun.krishnan@unsw.edu.au

Acknowledgements: A.K. was supported by a Career Development Award of the National Health and Medical Research Council of Australia (grant number 1065663). R.A was supported by an Early Career Post-Doctoral Fellowship of the National Health and Medical Research Council of Australia (\#1091006). MK was funded by Forefront, a collaborative research group supported from the National Health and Medical Research Council of Australia Program Grant (\#1037746).

This is the author manuscript accepted for publication and has undergone full peer review but has not been through the copyediting, typesetting, pagination and proofreading process, which may lead to differences between this version and the Version of Record. Please cite this article as doi: 10.1111/imj.14114

This article is protected by copyright. All rights reserved. 
Word Count Abstract: 250

Word Count Main Text: 3650

\section{ABSTRACT (250wrds)}

In Australia approximately 1.7million adults have evidence of chronic kidney disease (CKD). This complex disease can result in a multitude of complications, including hyperkalaemia which common and well recognised. . The advent of new therapeutics aimed at lowering serum potassium have raised the possibility of optimising potassium control to enable greater use of renin-angiotensin-aldosterone system inhibitors in the management of CKD. Recent studies suggest that hyperkalaemia also has implications for peripheral neuropathy in CKD, a complication that substantially contributes to patient morbidity. This review examines evidence of the relationship between potassium and peripheral neuropathy with discussion of clinical implications. We searched PubMed for original and review articles using prespecified key words, clinical guidelines and population data. The major findings were that contemporary CKD cohorts demonstrate a high prevalence of peripheral neuropathy, even in stage 3-4 CKD, including those without diabetes. The severity of the problem has been emphasized by an ominous rise in foot complications and amputation rates in dialysis patients, highlighting the need for increased awareness of the condition in earlier stages of CKD and targeted treatment strategies. It is likely that the pathophysiology of peripheral neuropathy in CKD is multifaceted with potential influences from potassium, vascular abnormalities, diabetes, inflammation and unknown middle molecules. Despite these complexities, the relationship between potassium and nerve function in 
dialysis has been well established and recent research in stage 3-4 CKD suggests that assertive potassium control may improve neuromuscular outcomes in CKD. These small studies should be confirmed in large, multicenter settings.

Key words: chronic kidney disease, potassium, peripheral neuropathy, foot complications, treatment

\section{INTRODUCTION}

Approximately 1 in 10 Australian adults have clinical evidence of chronic kidney disease (CKD). CKD encompasses a continuum of disease classified using a five-stage system from mild kidney damage to end-stage kidney disease (ESKD: Box 1). The prevalence of CKD is increasing due to an ageing population and growing rates of diabetes, hypertension and obesity. ${ }^{1}$ Hyperkalaemia is a common clinical condition in CKD and has many causes such as impaired renal handling and excretion of potassium, acidosis, insulin resistance, use of medications such as renin-angiotensinaldosterone system inhibitors, and high dietary potassium intake. ${ }^{2}$ Recent pharmaceutical advances in potassium binding agents highlight the possibility of optimizing potassium control, rather than just avoidance of severe hyperkalaemia. This is an appealing notion as it may enable

This article is protected by copyright. All rights reserved. 
greater use of renin-angiotensin-aldosterone system (RAAS) inhibitors in the management of CKD.

In addition to the cardiovascular and disease management benefits, normalising potassium may also improve neuromuscular complications in CKD. Neuromuscular tissues are excitable and recent studies have demonstrated that abnormal potassium homeostasis also has a detrimental effect on associated complications such as peripheral neuropathy and skeletal muscle function in CKD. ${ }^{3-8}$ Peripheral neuropathy is the most common neurological complication of CKD. It contributes to patient morbidity through pain, sensory loss and muscle weakness and is a key risk factor for foot complications. Peripheral neuropathy affects $60-90 \%$ of dialysis patients and has long been recognized in patients with advanced CKD. ${ }^{5,9}$ More recently, both population and CKD specific studies have demonstrated evidence of peripheral neuropathy in earlier stages of CKD. ${ }^{10-13}$ Given the prevalence and impact of peripheral neuropathy in CKD there is a pressing need for targeted treatment strategies. Recent research is emerging that suggests optimising potassium control including in patients before development of ESKD may represent one such strategy to improve neuromuscular outcomes in CKD.

To develop an evidence-based review of neuromuscular complications in CKD and the clinical implications of potassium control, we searched PubMed for original and review articles published using key words: chronic kidney disease, potassium, neuropathy and neuromuscular 
complications. We also sourced guidelines from KDOQI and population data from the Australian Bureau of Statistics.

\section{HYPERKALAEMIA AND KIDNEY DISEASE}

Hyperkalaemia is a classical and frequent complication of CKD. The most well recognised implications are its association with cardiac arrhythmia and the subsequent limitations in prescribing medications that potentially exacerbate hyperkalaemia. The incidence of hyperkalaemia increases with declining kidney function and CKD patients are therefore particularly predisposed to its occurrence. ${ }^{14}$ When defined as a serum potassium concentration $>5.5 \mathrm{mmol} / \mathrm{L}$, an episode of hyperkalaemia is experienced at least once per year in approximately $20 \%$ of patients with GFR-stage 3 CKD, $42 \%$ of patients with stage 4 CKD and $57 \%$ of patients with stage 5 CKD. ${ }^{14}$ Hypokalaemia (potassium $<3.5 \mathrm{mmol} / \mathrm{L}$ ) may also occur, though it is less frequent $(1-3 \%)$.

In health, potassium levels are tightly maintained between 3.5 and $5.0 \mathrm{mmol} / \mathrm{L}$ and large deviations from this range are potentially lethal. ${ }^{15}$ In CKD the factors contributing to hyperkalaemia in CKD may be intrinsic or extrinsic. Intrinsic factors are related to the disease and metabolic derangements. ${ }^{15}$ Progressive CKD is marked by a blunting of aldosteroneindependent potassium excretion that typically follows a potassium load. This mechanism is particularly important in patients with diabetes. In oliguric patients, reduced delivery of sodium and water to the distal potassium secretory sites of the nephron may also play a role in impaired 
potassium excretion. Additionally, given the loss of functional reserve, patients with CKD are particularly vulnerable to the extra-renal factors that influence the intra-and extra-cellular potassium gradient including acidosis and relative insulin deficiency. Extrinsic factors are related to medications and dietary intake. Hyperkalaemia is frequently induced by medications that are clinically indicated patients with CKD such as renin-angiotensin- aldosterone system (RAAS) inhibitors including angiotensin converting enzyme inhibitors (ACEis), angiotensin receptor blockers (ARBs), and mineralocorticoid receptor antagonists. ${ }^{16}$ These medications mainly impair renal excretion of potassium, but can increase serum potassium even in anuric patients through gastrointestinal effects. The use of two RAAS inhibitors simultaneously has been repeatedly shown to increase the incidence of hyperkalaemia. ${ }^{16}$

\section{MANAGEMENT OF CHRONIC HYPERKALAEMIA IN CKD}

Dietary modifications and the use of cation exchange resins are often used to address hyperkalaemia. ${ }^{15}$ In advanced CKD dietary potassium restriction is typically advocated if serum potassium exceeds 5mmol/L, despite few randomised studies assessing the approach. Evaluating these approaches in advanced CKD is challenging as food diaries may be inaccurate while the upregulation of gastrointestinal potassium excretion that occurs with advancing CKD and extracorporeal removal of potassium by dialysis renders urinary excretion a poor surrogate of oral intake. However, diets high in fruits and vegetables can reduce dietary acid load and may be beneficial for patients with advanced CKD. ${ }^{15}$ Therefore, restricting potassium without excluding 
healthy foods may be challenging. Moreover, due to the high variability in diets, particularly in multicultural communities, and the marked increase in manufactured foods available in recent decades, set dietary protocols (i.e. meal plans) may not be successful. Thus, intervention may require regular dietitian consultation, counselling and support with motivational interviewing, feedback and collaborative problem solving.

If other strategies are unsuccessful, cation exchange resins may be required. Sodium polystyrene sulfonate (SPS, Resonium A) exchanges sodium for potassium in the gastrointestinal lumen and has been available for the management of acute hyperkalaemia for many decades. In the acute setting small, uncontrolled studies suggest that SPS administration may reduce serum potassium by approximately $0.7-1.1 \mathrm{mmol} / \mathrm{L} \cdot{ }^{17}$ These studies do not exclude the effect of other concurrently administered therapies including sorbitol. Of concern, there are reports of fatal intestinal necrosis both with and without concurrent use of sorbitol. ${ }^{15}$

Two potential alternatives to SPS have recently emerged, patiromer calcium (RLY5016) and sodium zirconium cyclosilicate (ZS-9). ${ }^{2}$ Patiromer has been evaluated in short term studies of CKD patients treated with RAAS inhibitors and in a longer 52-week phase 2 study of patients with diabetes and $\mathrm{CKD} .{ }^{18-20}$ Patiromer appears to be generally well tolerated, with major adverse effects consisting of constipation, hypomagnesaemia and hypokalaemia. ${ }^{18-20}$ ZS-9 is another cation exchanger which is reported to have greater selectivity for monovalent cations than divalent cations (eg, calcium and magnesium) due to its crystalline lattice structure. It has been 
evaluated in two controlled studies ${ }^{21,22}$ and in a single study of open label use in severe hyperkalaemia. ${ }^{23}$

Strategies to optimize potassium control in CKD are appealing especially in the context of maximising RAAS blockade. ${ }^{15}$ However, no long-term studies have specifically evaluated this approach and at present no potassium binders are subsidised by the Australian Pharmaceutical Benefits Scheme. While the clinical focus regarding potassium in CKD has been almost entirely cardiac, the effect of even relative hyperkalaemia on other excitable tissues such as nerves and skeletal muscle is becoming increasingly recognized. This includes recent evidence that dietary potassium restriction may ameliorate the progression of neuropathy in CKD. ${ }^{10}$

\section{NEUROMUSCULAR EFFECTS OF HYPERKALAEMIA}

In addition to the risk of cardiac arrhythmia, patients with severe hyperkalaemia may also be afflicted with ascending paralysis due to neuromuscular failure. This is because potassium is a critical participant in the events underlying excitation, contraction and conduction in all neuromuscular and vascular tissues. ${ }^{15}$

Hodgkin and Huxley’s 1963 Nobel Prize winning work demonstrated that excitable tissues, such as nerve and muscle, are particularly reliant on potassium for the precise maintenance of an electrochemical gradient. The events that underpin nerve impulse transmission require a carefully maintained chemical and electrical concentration gradient or membrane potential which

This article is protected by copyright. All rights reserved. 
is partially determined by intracellular and extracellular potassium concentrations creating an electrical and chemical charge across the cell membrane. At rest an unequal distribution of $\mathrm{Na}^{+}$, $\mathrm{K}^{+}$and $\mathrm{Cl}^{-}$ions creates a net excess of negative charge on the inside of the cell and thus the resting membrane potential usually ranges from $-60 \mathrm{mV}$ to $-70 \mathrm{mV}$. If the membrane potential becomes more positive than the resting value it is termed depolarised, and alternatively, if the membrane potential is more negative than the resting value it is termed hyperpolarised. Hyperkalaemia shifts this critical gradient and causes the membrane potential to become depolarised which impairs function and homeostasis in excitable tissues.

Additionally, evidence suggests that the peripheral nerves are markedly susceptible to variation in steady state serum potassium levels ${ }^{7}$ as the blood-nerve barrier, which separates the blood and endoneurial environment, provides only a relatively non-selective diffusion barrier to small ions. $^{24}$

Peripheral nerves facilitate perception and interaction with the surrounding environment by conducting information to and from the central nervous system. As such, development of peripheral neuropathy disturbs the throughput of information and can have debilitating effects on quality of life and physical function. Peripheral neuropathy in the general population, irrespective of cause, has been shown to result in substantial symptom burden with pain, dysesthesia and loss of sensation as well as functional impairments including weakness, abnormalities of gait, a greater likelihood of falls and fall related injury as well as difficulty in 
activities of daily living. ${ }^{25}$ This condition is frequently caused by toxic and metabolic disturbances such as diabetes and CKD.

\section{IMPLICATIONS OF PERIPHERAL NEUROPATHY IN CKD}

Peripheral neuropathy in CKD has historically been considered a complication that becomes apparent when the estimated glomerular filtration rate (eGFR) falls below $12 \mathrm{~mL} / \mathrm{min} / 1.73 \mathrm{~m}^{2}$ and thus exclusively affecting patients with ESKD. While the progressive worsening of peripheral neuropathy with declining kidney function is well recognized, the prevalence of peripheral neuropathy in earlier stages of CKD has not been systemically investigated. Studies in contemporary CKD cohorts must contend with the increase in patients with co-existing diabetes, also a cause of peripheral neuropathy. Regardless, there is increasing evidence of demonstrable peripheral neuropathy related to CKD in contemporary pre-dialysis CKD and population-based cohorts. Two small studies have recently shown that peripheral neuropathy is present in $70 \%$ GFR-stage 3 and 4 CKD cohorts, of which one excluded patients with diabetes. ${ }^{10,11}$ Further evidence of the impact of CKD in the pathogenesis of peripheral neuropathy was recently highlighted in a population based cohort of 908 people that identified evidence of kidney disease as an independent risk factor for abnormal lower limb nerve conduction results. ${ }^{12}$ Similarly, the Health, Aging and Body Composition Study highlighted that age related peripheral nerve impairments were more common in elderly patients with CKD than those without, even after adjustment for diabetes. ${ }^{13}$ These studies in contemporary cohorts highlight that there is objective

This article is protected by copyright. All rights reserved. 
evidence of peripheral neuropathy at milder stages of CKD than historically recognized and that even in patients with diabetes, the coexistence of CKD is an important contributor to neuropathy. This recognition may provide a window of opportunity for early identification and management (Box 2). The clinical implications of poorly managed peripheral neuropathy are extensive. At its most severe, the presence of peripheral neuropathy contributes as a key risk factor for foot lesions, ulceration and amputation in diabetic and CKD patients alike (Fig 1). ${ }^{26}$ Amputation rates in diabetic dialysis patients are 10 fold higher than the general diabetic population and 1 year post-surgery mortality rates of $50 \%$ have been reported. ${ }^{27}$ As such, early recognition of neuropathy and foot complications in CKD are critical to interrupt this cascade of events.

Beyond foot lesions and their sequelae, peripheral neuropathy can have a debilitating effect on quality of life and physical function ${ }^{25}$ a relationship that is also evident in dialysis patients. ${ }^{28,29}$ In one study of 301 dialysis patients, ${ }^{29}$ neuromuscular symptoms were independently associated with reduced self-reported physical function and increased depressive symptoms. Another study reported a significant reduction in gait speed that was negatively correlated with severity of peripheral neuropathy and greater difficulty in ADLs. ${ }^{28}$

Even prior to renal replacement therapy patients with CKD have reduced physical activity and increased disability compared to those without. ${ }^{30}$ Physical limitation in CKD is often attributed to cardiovascular complications, sarcopenia and metabolic disturbances, but these factors may not fully explain such disability. An inverse relationship between peripheral neuropathy and 
objective markers of lower limb function such as walking speed have been shown in the general population ${ }^{25}$ and in end-stage kidney disease. ${ }^{28}$ Although not systematically examined, it seems likely that neuropathy may also lead to significant functional impairment in moderate CKD. ${ }^{10,13}$

In terms of treatment, painful symptoms of peripheral neuropathy can be managed using a range of tricyclic anti-depressants or anticonvulsants, which may have complicated dosing restrictions in $\mathrm{CKD} .{ }^{27}$ Although these provide symptomatic relief, they do not halt the progression of neuropathy. Renal transplantation may improve neuropathy. ${ }^{9}$ However, the use of calcineurin inhibitors within immunosuppressive regimens or development of new-onset diabetes after transplantation may hinder improvements, ${ }^{31}$ demonstrating the need for preventative strategies. The impact of peripheral neuropathy on the immediate and continuing cost of disease management emphasizes the need for targeted treatment strategies prior to the severe manifestations that may occur in dialysis.

\section{THE PATHOPHYSIOLOGY OF PERIPHERAL NEUROPATHY IN CKD}

Peripheral neuropathy in CKD or 'uraemic neuropathy' was first described in 1961. Despite the advent of renal replacement therapy and decades of advancements in dialysis technology this complication still affects $60-90 \%$ of ESKD patients. ${ }^{5,9}$ In CKD, there are many pathological exposures that may contribute to peripheral neuropathy such as amyloid, vasculitis, small vessel disease and diabetes, to name a few. In depth analysis of all of these factors is beyond the scope of this review. However, examination of modifiable factors among this milieu may provide a 
feasible treatment strategy for neuropathy despite the complexity of its pathophysiology and will be the focus of this paper.

Initial pathophysiological descriptions were based on observational studies with the use of early renal replacement therapies. The concept that uremic neuropathy may be caused by a dialyzable neurotoxin, or toxins, was drawn from associations between reduced neuropathy severity and increasing dialysis frequency and efficiency. A popular early hypothesis was the 'middle molecule' hypothesis. ${ }^{32}$ This was based the relationship between reduced neuropathy severity and peritoneal dialysis, despite higher urea and creatinine levels than patients on haemodialysis. ${ }^{32}$ In that era, peritoneal dialysis achieved better clearance of molecules in the middle molecular weight range (500-2000 Daltons) and thus the prevailing hypothesis was that one or more uremic toxins in this range were responsible for uremic neuropathy. ${ }^{32}$ The definition of middle molecules has been expanded to incorporate molecules that are not well removed by dialysis for other reasons including binding moieties and multicompartmental behavior. ${ }^{33}$ While the wide range and ambiguity of the middle molecule hypothesis has made it difficult to gain information on specific candidates for targeted therapies in uraemic neuropathy, one study has demonstrated the superiority of large pore dialysis membranes in prevention of peripheral neuropathy. ${ }^{34}$ Several of these broad-definition substrates such as cytokines and advanced glycation end products are likely to adversely affect peripheral nerve homeostasis via inflammation and vascular damage. ${ }^{27}$ Additionally, some evidence suggests parathyroid hormone 
and $\beta 2$ microglobulin may be involved in the pathogenesis of peripheral neuropathy ${ }^{35,36}$ though direct evidence demonstrating neurotoxicity is limited or conflicting. ${ }^{37,38}$

Another potential candidate known to accumulate in kidney failure is potassium. ${ }^{39}$ While potassium does not fall into the classic definition of middle molecule, it does exhibit multicompartmental behaviour. Initial studies implicating potassium as a neurotoxin were conducted in dialysis patients using specialised electrophysiological techniques. Beginning in 2002, a series of studies demonstrated evidence of significant membrane depolarisation prior to dialysis that was almost entirely normalised immediately after dialysis. ${ }^{5-7}$ Analysis of serum biochemistry demonstrated significant correlations specifically between serum potassium and pre-dialysis measures of membrane potential. ${ }^{7}$ In total, these studies demonstrated a generalised, systemic effect of high serum potassium concentrations on the membrane potential of motor, sensory, upper and lower limb peripheral nerves. ${ }^{5-7}$ The depolarising effects of potassium were also demonstrated in muscle fibres of dialysis patients. Potassium was strongly correlated with muscle membrane depolarisation which was partially normalised by dialysis only to rebound 1 hour after dialysis. ${ }^{8}$

In order to rule out the possible influence of other dialyzable metabolites, these studies were followed up by a dialysis intervention study. ${ }^{3}$ The intervention involved maintaining serum potassium levels at $>5 \mathrm{mmol} / \mathrm{L}$ for the first 3 hours of a modified dialysis session, whilst all other solutes were removed as per normal dialysis. Despite significant clearance of other uraemic 
toxins, measures of membrane depolarisation persisted whilst potassium was elevated and only improved following reduction of potassium in the subsequent dialysis period with reduced potassium in the dialysate. Importantly, the extent of membrane depolarisation seen in the dialysis patients was significantly greater than expected for healthy axons mathematically modelled at similar serum potassium suggesting structural as well as functional damage. ${ }^{3}$ Maintenance of ionic gradients and the resting membrane potential are critical to normal neuronal homeostasis and function. Molecular studies have suggested that prolonged loss of homeostasis may lead to altered sodium channel activity, mitochondrial compromise and, in turn, reverse operation of the sodium/calcium exchanger leading to axonal degeneration. ${ }^{40}$

Recent advances in ultrasound technology have demonstrated that the functional changes observed in electrophysiological studies are accompanied by structural and vascular abnormalities. One study demonstrated that the median nerve in haemodialysis patients has a greater cross sectional area and greater hypoechoic features suggesting enlargement that may be caused by intraneural oedema. ${ }^{4}$ Ultrasound measurements using Doppler sonograms have also detected intraneural blood flow in dialysis patients, which is a pathological feature not detectable in controls. ${ }^{41}$ These abnormalities of structure were again significantly reduced following dialysis and the improvements in nerve cross-sectional area across a dialysis session correlated significantly with the degree of potassium removal. ${ }^{4,41}$ It has been hypothesized that these morphological changes could be induced by swelling at the node of Ranvier, myelin sheath and periaxonal space, mediated by the abnormal passage of ions into the Schwann cell as it attempts 
to buffer excess potassium which leads movement of water into the cell causing swelling. 3,4 Taken together these, studies provide evidence for a potential link between changes in nerve structure and function induced by hyperkalaemia, which may ultimately underlie long-term disability due to neuropathy.

One difficulty in assessing the role of potassium in the pathogenesis of neuropathy is its fluctuating serum concentration. In particular, potassium removal is a key goal of dialysis and in patients with ESKD serum potassium levels may be normalised or even below normal for a portion of time. However, reduced serum potassium in patients treated with haemodialysis is a transient state that rapidly rebounds due to intracellular potassium release and thus serum potassium concentrations approach pre-dialysis values within six hours. ${ }^{42}$ Additionally, these fluctuations may cause metabolic stress to the nerve and generate pathological damage not directly attributable to hyperkalaemia per se. ${ }^{43}$ Another clinical context that challenges the potassium hypothesis is in peritoneal dialysis (PD) where patients typically demonstrate lower serum potassium than haemodialysis patients. ${ }^{44}$ There are very few and only small studies comparing neuropathy outcomes in modern haemodialysis compared to PD. ${ }^{45-48}$ Two of these studies focus on carpal tunnel syndrome and as an adjunct report no differences in peripheral neuropathy between groups. ${ }^{46,47}$ Another larger study demonstrated similar overall rates of clinically diagnosed peripheral neuropathy, though the PD group had significantly better nerve conduction study results in both upper and lower limb nerves which were attributed to greater residual renal function in the PD group. ${ }^{48}$ Finally, a small study investigating nerve function 
demonstrated improved nerve excitability indices in PD compared to haemodialysis, though the groups were matched for neuropathy severity making it unsuitable for evaluation of overt neuropathy comparisons. ${ }^{45}$ Overall, there is insufficient evidence to suggest that there are differences in peripheral neuropathy outcomes between PD and HD.

Given the complexities of potassium homeostasis in ESKD, the most compelling evidence for a role of hyperkalaemia in peripheral neuropathy of CKD possibly comes from a recent randomized controlled trial that examined a potassium reduction intervention over a two year period in patients with GFR stage 3-4 CKD. ${ }^{10}$ This study was undertaken in 47 patients with stages 3 and 4 CKD who were randomised to either control or an intervention of dietary potassium restriction with target potassium of $\leq 4.5 \mathrm{mmol} / \mathrm{L}$. Patients in the intervention had regular dietician consultation for strategies to reduce potassium intake and were prescribed SPS (Resonium A) if they could not achieve the target with dietary intervention alone. Neuropathy and physical function were assessed at six-monthly intervals for the 2 year trial duration. The intervention achieved a significant reduction in mean potassium levels in the intervention arm and in $53 \%$ of patients, this was achieved without the need for supplemental SPS. The primary outcome was change in total neuropathy score which was significantly reduced in the intervention compared to the control group over the 2 year duration of the study. Specifically, the intervention group had stable neuropathy, while the controls demonstrated constant progression, and at trial completion the intervention group had significantly reduced neuropathy severity compared to controls. Additionally, the intervention group demonstrated an improvement in 
walking speed highlighting the functional implications of neuropathy progression in CKD. These effects were present despite unrestricted nephrology care including the use of renin-angiotensinaldosterone system inhibitors and diuretics as clinically warranted. ${ }^{10}$ However, it is important to acknowledge that this was a small, single site, proof of concept RCT and thus sample size is a limitation. It was not sufficiently powered to examine the effect of diabetic status on the primary outcome. Nevertheless, this is an important preliminary step that needs to be replicated in a larger, multicentre setting.

\section{SUMMARY}

Elevated potassium, including overt hyperakalemia, is a common complication in CKD. Management of chronic hyperkalaemia is complex and consists mainly of avoiding potassiumsparing medications, dietary interventions, and potentially using gastrointestinal exchange resins. New agents with clearer safety profiles may soon enter the market. Despite their limitations, the present indication for these strategies is to treat or avoid overt hyperkalaemia and to reduce the risk of cardiac complications. Studies in the last fifteen years have identified potassium as a critical, modifiable element in the physiology of peripheral neuropathy in CKD. Additionally, evidence from contemporary cohorts have highlighted that peripheral neuropathy is evident prior to dialysis and may provide a window of opportunity to reduce the physical function and clinical complications associated with advanced peripheral neuropathy. Improved methods for lowering serum potassium may permit more assertive RAAS inhibition to prevent CKD progression and cardiovascular disease, in addition to preventing neuropathy. 


\section{REFERENCES}

1. Statistics ABo. Australian Health Survey: Biomedical Results for Chronic Diseases, 2011-12. In: ABS, (ed.). Canberra2013.

2. Ingelfinger JR. A New Era for the Treatment of Hyperkalemia? N Engl J Med. 2015; 372: 275-7.

3. Arnold R, Pussell BA, Howells J, et al. Evidence for a causal relationship between hyperkalaemia and axonal dysfunction in end-stage kidney disease. Clin Neurophysiol. 2014; 125: 179-85.

4. Borire AA, Arnold R, Pussell BA, et al. Haemodialysis alters peripheral nerve morphology in endstage kidney disease. Clin Neurophysiol. 2017; 128: 281-6.

5. Krishnan AV, Phoon RK, Pussell BA, Charlesworth JA, Bostock H and Kiernan MC. Altered motor nerve excitability in end-stage kidney disease. Brain. 2005; 128: 2164-74.

6. Krishnan AV, Phoon RK, Pussell BA, Charlesworth JA and Kiernan MC. Sensory nerve excitability and neuropathy in end stage kidney disease. J Neurol Neurosurg Psychiatry. 2006; 77: 548-51.

7. Kiernan MC, Walters RJ, Andersen KV, Taube D, Murray NM and Bostock H. Nerve excitability changes in chronic renal failure indicate membrane depolarization due to hyperkalaemia. Brain. 2002; 125: 1366-78.

8. Z'Graggen WJ, Aregger F, Farese $S$, et al. Velocity recovery cycles of human muscle action potentials in chronic renal failure. Clin Neurophysiol. 2010; 121: 874-81.

9. Krishnan AV and Kiernan MC. Neurological complications of chronic kidney disease. Nat Rev Neurol. 2009; 5: 542-51.

10. Arnold R, Pianta TJ, Pussell BA, et al. Randomized, Controlled Trial of the Effect of Dietary Potassium Restriction on Nerve Function in CKD. Clinical journal of the American Society of Nephrology: CJASN. 2017; 12: 1569-77.

11. Aggarwal HK, Sood S, Jain D, Kaverappa V and Yadav S. Evaluation of spectrum of peripheral neuropathy in predialysis patients with chronic kidney disease. Ren Fail. 2013; 35: 1323-9.

12. Hanewinckel R, Ikram MA, Franco OH, Hofman A, Drenthen J and van Doorn PA. High body mass and kidney dysfunction relate to worse nerve function, even in adults without neuropathy. $J$ Peripher Nerv Syst. 2017; 22: 112-20.

13. Moorthi RN, Doshi S, Fried LF, et al. Chronic kidney disease and peripheral nerve function in the Health, Aging and Body Composition Study. Nephrol Dial Transplant. 2018.

14. Einhorn LM, Zhan M, Hsu VD, et al. The frequency of hyperkalemia and its significance in chronic kidney disease. Arch Intern Med. 2009; 169: 1156-62.

15. Fried L, Kovesdy CP and Palmer BF. New options for the management of chronic hyperkalemia. Kidney International Supplements. 2017; 7: 164-70.

16. Phillips CO, Kashani A, Ko DK, Francis G and Krumholz HM. Adverse effects of combination angiotensin II receptor blockers plus angiotensin-converting enzyme inhibitors for left ventricular dysfunction: a quantitative review of data from randomized clinical trials. Arch Intern Med. 2007; 167: 1930-6.

17. Fordjour KN, Walton T and Doran JJ. Management of hyperkalemia in hospitalized patients. Am J Med Sci. 2014; 347: 93-100. 
18. Bakris GL, Pitt B, Weir MR, et al. Effect of Patiromer on Serum Potassium Level in Patients With Hyperkalemia and Diabetic Kidney Disease: The AMETHYST-DN Randomized Clinical Trial. JAMA. 2015; 314: 151-61.

19. Weir MR, Bushinsky DA, Benton WW, et al. Effect of Patiromer on Hyperkalemia Recurrence in Older Chronic Kidney Disease Patients on Renin-Angiotensin-Aldosterone-System Inhibitors. Am J Med. 2017.

20. Weir MR, Bakris GL, Bushinsky DA, et al. Patiromer in patients with kidney disease and hyperkalemia receiving RAAS inhibitors. N Engl J Med. 2015; 372: 211-21.

21. Kosiborod M, Rasmussen HS, Lavin $\mathrm{P}$, et al. Effect of sodium zirconium cyclosilicate on potassium lowering for 28 days among outpatients with hyperkalemia: the HARMONIZE randomized clinical trial. JAMA. 2014; 312: 2223-33.

22. Packham DK, Rasmussen HS, Lavin PT, et al. Sodium zirconium cyclosilicate in hyperkalemia. $N$ Engl J Med. 2015; 372: 222-31.

23. Kosiborod M, Peacock WF and Packham DK. Sodium zirconium cyclosilicate for urgent therapy of severe hyperkalemia. N Engl J Med. 2015; 372: 1577-8.

24. Weerasuriya A. Permeability of endoneurial capillaries to $\mathrm{K}, \mathrm{Na}$ and $\mathrm{Cl}$ and its relation to peripheral nerve excitability. Brain Res. 1987; 419: 188-96.

25. Hanewinckel R, Drenthen J, Verlinden VJA, et al. Polyneuropathy relates to impairment in daily activities, worse gait, and fall-related injuries. Neurology. 2017; 89: 76-83.

26. Kaminski MR, Raspovic A, McMahon LP, et al. Risk factors for foot ulceration and lower extremity amputation in adults with end-stage renal disease on dialysis: a systematic review and metaanalysis. Nephrol Dial Transplant. 2015; 30: 1747-66.

27. Pop-Busui R, Roberts L, Pennathur S, Kretzler M, Brosius FC and Feldman EL. The management of diabetic neuropathy in CKD. Am J Kidney Dis. 2010; 55: 365-85.

28. Jin SH, Park YS, Park YH, Chang HJ and Kim SR. Comparison of Gait Speed and Peripheral Nerve Function Between Chronic Kidney Disease Patients With and Without Diabetes. Annals of rehabilitation medicine. 2017; 41: 72-9.

29. Amro A, Waldum B, Dammen T, Miaskowski C and Os I. Symptom clusters in patients on dialysis and their association with quality-of-life outcomes. Journal of renal care. 2014; 40: 23-33.

30. White SL, Dunstan DW, Polkinghorne KR, Atkins RC, Cass A and Chadban SJ. Physical inactivity and chronic kidney disease in Australian adults: The AusDiab study. Nutrition, Metabolism and Cardiovascular Diseases. 2011; 21: 104-12.

31. Arnold R, Pussell BA, Pianta TJ, Lin CS, Kiernan MC and Krishnan AV. Association between calcineurin inhibitor treatment and peripheral nerve dysfunction in renal transplant recipients. American journal of transplantation : official journal of the American Society of Transplantation and the American Society of Transplant Surgeons. 2013; 13: 2426-32.

32. Babb AL, Ahmad S, Bergstrom J and Scribner BH. The middle molecule hypothesis in perspective. Am J Kidney Dis. 1981; 1: 46-50.

33. Vanholder RC, Glorieux GL and De Smet RV. Back to the future: middle molecules, high flux membranes, and optimal dialysis. Hemodialysis international International Symposium on Home Hemodialysis. 2003; 7: 52-7. 
34. Malberti F, Surian M, Farina M, et al. Effect of hemodialysis and hemodiafiltration on uremic neuropathy. Blood Purification. 1991; 9: 285-95.

35. Massry SG. Parathyroid hormone: a uremic toxin. Adv Exp Med Biol. 1987; 223: 1-17.

36. Goldstein DA, Chui LA and Massry SG. Effect of parathyroid hormone and uremia on peripheral nerve calcium and motor nerve conduction velocity. J Clin Invest. 1978; 62: 88-93.

37. Avram MM, Feinfeld DA and Huatuco AH. Search for the uremic toxin. Decreased motor-nerve conduction velocity and elevated parathyroid hormone in uremia. N Engl J Med. 1978; 298: 1000-3.

38. Schaefer K, Offermann G, von Herrath D, Schroter R, Stolzel R and Arntz HR. Failure to show a correlation between serum parathyroid hormone, nerve conduction velocity and serum lipids in hemodialysis patients. Clin Nephrol. 1980; 14: 81-8.

39. Bostock H, Walters RJ, Andersen KV, Murray NM, Taube D and Kiernan MC. Has potassium been prematurely discarded as a contributing factor to the development of uraemic neuropathy? Nephrol Dial Transplant. 2004; 19: 1054-7.

40. Persson AK, Hoeijmakers JG, Estacion M, Black JA and Waxman SG. Sodium Channels, Mitochondria, and Axonal Degeneration in Peripheral Neuropathy. Trends in molecular medicine. 2016; 22: 377-90.

41. Borire AA, Arnold R, Pussell BA, et al. Effects of hemodialysis on intraneural blood flow in endstage kidney disease. Muscle Nerve. 2017.

42. Blumberg A, Roser HW, Zehnder C and Muller-Brand J. Plasma potassium in patients with terminal renal failure during and after haemodialysis; relationship with dialytic potassium removal and total body potassium. Nephrol Dial Transplant. 1997; 12: 1629-34.

43. Krishnan AV, Phoon RK, Pussell BA, Charlesworth JA, Bostock $\mathrm{H}$ and Kiernan MC. Ischaemia induces paradoxical changes in axonal excitability in end-stage kidney disease. Brain. 2006; 129: 1585-

92.

44. Torlen K, Kalantar-Zadeh K, Molnar MZ, Vashistha T and Mehrotra R. Serum potassium and cause-specific mortality in a large peritoneal dialysis cohort. Clinical journal of the American Society of Nephrology : CJASN. 2012; 7: 1272-84.

45. Arnold R, Pussell BA, Kiernan MC and Krishnan AV. Comparative study to evaluate the effects of peritoneal and hemodialysis on peripheral nerve function. Muscle Nerve. 2016; 54: 58-64.

46. Kwon HK, Pyun SB, Cho WY and Boo CS. Carpal tunnel syndrome and peripheral polyneuropathy in patients with end stage kidney disease. J Korean Med Sci. 2011; 26: 1227-30.

47. Bicknell JM, Lim AC, Raroque HG, Jr. and Tzamaloukas AH. Carpal tunnel syndrome, subclinical median mononeuropathy, and peripheral polyneuropathy: common early complications of chronic peritoneal dialysis and hemodialysis. Arch Phys Med Rehabil. 1991; 72: 378-81.

48. Janda K, Stompor T, Gryz E, et al. [Evaluation of polyneuropathy severity in chronic renal failure patients on continuous ambulatory peritoneal dialysis or on maintenance hemodialysis]. Przegl Lek. 2007; 64: 423-30.

49. KDIGO. Summary of Recommendation Statements. Kidney International Supplements. 2013; 3: $5-14$.

50. National Kidney Foundation. K/DOQI clinical practice guidelines for chronic kidney disease: evaluation, classification, and stratification. Am J Kidney Dis. 2002; 39: S1-266. 


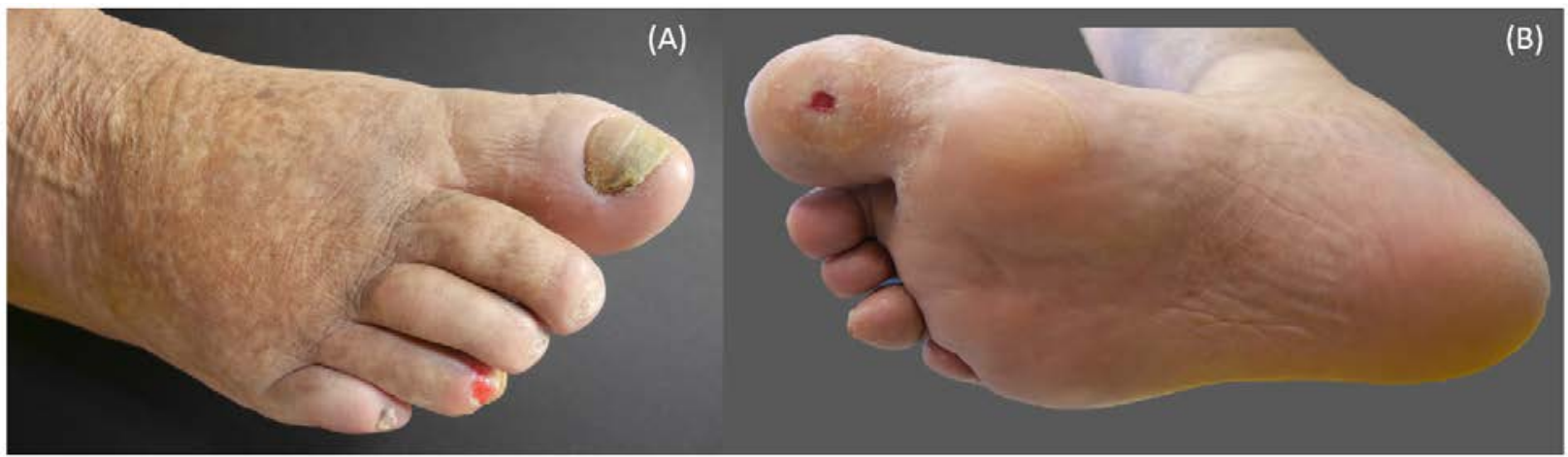

Figure 1. Case of severe peripheral neuropathy and associated foot complications in patient with diabetic-CKD. Panel A depicts neuropathic features of trophic skin and poor nail quality with lesion on $4^{\text {th }}$ toe. Visualization of intrinsic foot muscles is confounded by oedema. Panel B plantar aspect demonstrates lesion on $1^{\text {st }}$ toe, pressure areas under $1^{\text {st }}$ and $5^{\text {th }}$ metatarsal heads and toe curling, which may occur due to denervation of intrinsic foot muscles. Patient reports no feeling upto the knees and $\mathrm{x}$-rays demonstrated mild 1st/2nd tarsometatarsal and tibiotalar joint degeneration as well as focal plantar ligament ossification, consistent with a neuropathic arthropathy.

Box 1. Five Stage Classification of Chronic Kidney

Disease *

GFR Stage

G1 Evidence of kidney damage with normal eGFR $>90 \mathrm{~mL} / \mathrm{min} / 1.73 \mathrm{~m}^{2}$

G2 Evidence of kidney damage with mild reduction of eGFR $60-89 \mathrm{~mL} / \mathrm{min} / 1.73 \mathrm{~m}^{2}$

G3a Mild to moderately reduced eGFR $45-59 \mathrm{~mL} / \mathrm{min} / 1.73 \mathrm{~m}^{2}$

G3b Moderate to severely reduced eGFR

$30-44 \mathrm{~mL} / \mathrm{min} / 1.73 \mathrm{~m}^{2}$

G4 Severely reduced eGFR $15-29 \mathrm{~mL} / \mathrm{min} / 1.73 \mathrm{~m}^{2}$

G5 Renal failure or dialysis eGFR $<15 \mathrm{~mL} / \mathrm{min} / 1.73 \mathrm{~m}^{2}$ 
Classification as defined by the KDIGO. Summary of recommendation statements ${ }^{49}$ updating KDOQI Clinical Practice Guidelines ${ }^{50}$

*Staging of G1-3a CKD is also influenced by the degree of albuminuria (albumin excretion rate) A1: $<30 \mathrm{mg} /$ day; A2: 30 - $300 \mathrm{mg} /$ day; A3: $>300 \mathrm{mg} /$ day $^{49}$

This article is protected by copyright. All rights reserved. 


\begin{tabular}{|c|c|c|c|}
\hline & Characteristic features & Patient symptoms & Clinical signs \\
\hline Early features & $\begin{array}{l}\text { Insidious onset } \\
\text { Preferentially involves } \\
\text { distal nerves } \\
\text { More severe in lower } \\
\text { limbs than upper limbs } \\
\text { Symmetrical } \\
\text { Slowly progressive }\end{array}$ & $\begin{array}{l}\text { Confined to lower } \\
\text { limbs } \\
\text { Numbness } \\
\text { Paraesthesia } \\
\text { Lancinating pain }\end{array}$ & $\begin{array}{l}\text { Confined to lower limbs } \\
\text { most apparent distally. } \\
\text { Distal sensory loss to } \\
\text { pinprick and vibration } \\
\text { Reduced or absent ankle } \\
\text { deep tendon reflexes } \\
\text { Reduced amplitude or } \\
\text { absence of lower limb } \\
\text { sensory nerve conduction } \\
\text { studies }\end{array}$ \\
\hline $\begin{array}{l}\text { Advanced } \\
\text { cases }\end{array}$ & $\begin{array}{l}\text { Slowly progressive } \\
\text { Symptoms in legs extend } \\
\text { to knee, upper limb } \\
\text { involvement may } \\
\text { become apparent } \\
\text { Evidence of motor } \\
\text { involvement }\end{array}$ & $\begin{array}{l}\text { Insensate feet } \\
\text { Paraesthesia in } \\
\text { fingers } \\
\text { Weakness, } \\
\text { clumsiness walking } \\
\text { Balance disturbances }\end{array}$ & $\begin{array}{l}\text { Distal sensory loss } \\
\text { extending into leg and in } \\
\text { distal hands/fingers } \\
\text { Absent ankle deep tendon } \\
\text { reflexes and reduced at } \\
\text { knee } \\
\text { Absent lower limb sensory } \\
\text { and possibly motor nerve } \\
\text { conduction studies }\end{array}$ \\
\hline $\begin{array}{l}\text { Co-existing } \\
\text { Diabetes }\end{array}$ & $\begin{array}{l}\text { Typically evident at } \\
\text { earlier stages of CKD } \\
\text { and more severe at end- } \\
\text { stage CKD } \\
\text { Presentation and } \\
\text { symptoms - similar to } \\
\text { peripheral neuropathy in } \\
\text { CKD alone } \\
\text { Progression may be more } \\
\text { rapid }\end{array}$ & $\begin{array}{l}\text { Increased patient } \\
\text { awareness of } \\
\text { condition } \\
\text { Possible } \\
\text { predominance of } \\
\text { painful symptoms in } \\
\text { early stages } \\
\text { Advanced motor } \\
\text { disturbances and risk } \\
\text { of foot } \\
\text { complications with } \\
\text { progression }\end{array}$ & $\begin{array}{l}\text { Distal sensory loss or pain } \\
\text { in feet } \\
\text { Addition or predominance } \\
\text { of small fibre features eg. } \\
\text { severe burning/shooting } \\
\text { pain, altered temperature } \\
\text { and pain perception, } \\
\text { hyperalgesia or allodynia }\end{array}$ \\
\hline
\end{tabular}

This article is protected by copyright. All rights reserved. 


\section{University Library}

\section{- M M N E R VA A gateway to Melbourne's research publications}

Minerva Access is the Institutional Repository of The University of Melbourne

Author/s:

Arnold, R;Pianta, TJ;Pussell, BA;Endre, Z;Kiernan, MC;Krishnan, AV

Title:

Potassium control in chronic kidney disease: implications for neuromuscular function

Date:

2019-07-01

Citation:

Arnold, R., Pianta, T. J., Pussell, B. A., Endre, Z., Kiernan, M. C. \& Krishnan, A. V. (2019).

Potassium control in chronic kidney disease: implications for neuromuscular function.

INTERNAL MEDICINE JOURNAL, 49 (7), pp.817-825. https://doi.org/10.1111/imj.14114.

Persistent Link:

http://hdl.handle.net/11343/286106 